\title{
Chiral Vertex Operators in Off-Conformal Theory: The Sine-Gordon Example
}

\author{
Shau-Jin Chang \\ Department of Physics \\ University of Illinois at Urbana-Champaign \\ 1110 West Green Street \\ Urbana, Illinois 61801-3080 USA \\ R. Rajaramant \\ Department of Physics \\ University of Illinois at Urbana-Champaign \\ 1110 West Green Street \\ Urbana, Illinois 61801-3080 USA \\ and \\ Indian Institute of Science \\ Bangalore 560012, India
}

Typed by: Sandy Chancey/Char Cleek-Agans 


\begin{abstract}
We study chiral vertex operators in the sine-Gordon $[\mathrm{SG}]$ theory, viewed as an off-conformal system. We find that these operators, which would have been primary fields in the conformal limit, have interesting and, in some ways, unexpected properties in the SG model. Some of them continue to have scale- invariant dynamics even in the presence of the non-conformal cosine interaction. For instance, it is shown that the Mandelstam operator for the bosonic representation of the Fermi field does not develop a mass term in the SG theory, contrary to what the real Fermi field in the massive Thirring model is expected to do. It is also shown that in the presence of the non-conformal interactions, some vertex operators have unique Lorentz spins, while others do not.
\end{abstract}




\section{INTRODUCTION}

In this paper, we derive some interesting properties of vertex functions in the quantum sineGordon [SG] model. This model has been under study for a long time. Its several remarkable properties, such as its integrability and exact solubility, the presence of soliton solutions and topological sectors, the availability of its exact S-matrix etc, have been known since the seventies. In this paper, however, our interest derives from a somewhat different feature of the SG model, namely, that it can be viewed as a very attractive example of an off-conformal system. In recent years there has been considerable interest in studying off-critical (off-conformal) theories by techniques of conformal perturbation theory. In particular, Zamalodchikov showed that for certain off-conformal theories, new conserved currents could be found through such methods. ${ }^{1}$ Subsequently Bernard and LeClair applied these methods to the SG system viewed as an off-conformal theory. ${ }^{2}$ They derived new nonlocal currents for this system which obey a very interesting algebraic and braiding properties, powerful enough to lead to its exact S- matrix. The fermionic version of these currents was also identified. ${ }^{3}$

Our interest in this area was triggered by the Bernard-LeClair work. They had identified their new conserved currents for the SG model within the conformal perturbation theory (CPT) framework. We were interested in seeing whether such results obtained in the CPT framework also hold, literally in the same form, under conventional canonical quantization in familiar Minkowskian time.

We showed in an earlier paper that in fact they don't, and require modification. ${ }^{4}$ Specifically, we found exact expressions for the SG nonlocal currents which were similar to, but not precisely the same as those quoted by Bernard and LeClair. The reason for this discrepancy is presumably because in CPT, fields are schematically denoted by their deep ultra-violet behavior, rather than their actual behavior in all of space-time in the off- conformal system. We also found that Zamolodchikov's argument for the strict absence of higher order corrections to these nonlocal currents manifests itself in the canonical framework through the fact that certain vertex operators commute with the interaction. 
Such results motivated us to study more systematically in this paper, the properties of general chiral vertex functions in the SG system, under canonical quantization. Recall that in the absence of the cosine interaction, the SG system is a free massless scalar field, which is the prototype example of a conformally invariant field theory, with a pair of chiral fields which are respectively left and right moving. Chiral vertex operators, which are exponentials of these chiral fields, play a major role in that conformal theory by providing an infinite number of primary fields, each characterized by a different pair of Lorentz spin and scale dimensions. We wanted to show how the behavior of these vertex operators under time-evolution, Lorentz boost and scaling, is affected by the presence of the non-conformal $-\lambda(\cos \beta \phi)$ interaction in the SG theory.

We find several interesting results. Chiefly,

(i) There exist families of local vertex operators which commute with the interaction potential of the SG theory, for $\beta^{2}<1$. They therefore evolve in time in a scale invariant fashion, as if this non-conformal interaction were simply not there.

(ii) A particularly interesting example of this family is the Mandelstam construction of the Fermi field as part of the bosonization programm. ${ }^{5,6}$ This Mandelstam operator in fact turns out not to contain a mass term in its equation of motion for $\beta^{2}<1$, in contrast to what equivalence to the massive Thirring model would lead us to expect. We find that it is only when $\beta=1$, which corresponds to free fermions, that the Mandelstam operator's dynamics has a finite mass term.

(iii) We find that, the Lorentz spin of vertex operators is affected by the presence of the interaction term. Unlike in the conformal limit, the vertex operators here do not carry a unique Lorentz spin. Special subsets of them which do, are identified.

(iv) Surprisingly, despite the presence of the scale-breaking interaction, the vertex operators continue to be eigenfunctions of the scaling operator at any given instant.

Even though all our results are obtained only for the SG theory, we believe that our analysis carries useful lessons about off-conformal theories in general.

This paper is structured as follows: In sec. 2, we gather together basic equal-time commutators of the chiral fields, and define chiral vertex operators, keeping track of potential ultraviolet and infra-red divergences of this $(1+1)$ dimensional theory. Some inputs for this section are detailed 
in Appendix A. In sec. 3, we show that certain classes of vertex operators commute with the interaction, and discuss the significance of this fact to the nonlocal conserved currents obtained in

ref. 4. In sec. 4, we point out how for the above classes of vertex functions, which include the Mandelstam operator, their dynamics is scale invariant. In sec. 5, the scaling and Lorentz boost behavior of vertex operators is discussed.

\section{PRELIMINARIES}

We will treat the sine-Gordon [SG] system using the canonical equal-time algebra of chiral fields and their vertex functions. Much of conformal field theoretic literature deals with such fields using operator product expansions in terms of complex Euclidean coordinates. Equal-time Minkowskian commutators for chiral fields are less familiar. Therefore for completeness we will gather together in this section a few basic equal-time formulae needed for our calculations in the subsequent sections.

Consider a relativistic scalar field $\phi(x, t)$ in $1+1$ dimensions governed by a Lagrangian density of the form

$$
\mathcal{L}=\frac{1}{8 \pi} \dot{\phi}^{2}-\frac{1}{8 \pi}\left(\frac{\partial \phi}{\partial x}\right)^{2}-V(\phi)
$$

where dot refers to time-derivative. For the SG theory, the classical potential is

$$
V(\phi)=-\lambda \cos \beta \phi
$$

Let us define "chiral" fields $\rho$ and $\bar{\rho}$ by

$$
\begin{aligned}
\rho(x, t) & \equiv \frac{1}{2}\left[\phi(x, t)+\int_{-\infty}^{x} \dot{\phi}(y, t) d y\right] \\
\bar{\rho}(x, t) & \equiv \frac{1}{2}\left[\phi(x, t)-\int_{-\infty}^{x} \dot{\phi}(y, t) d y\right]
\end{aligned}
$$

Clearly $\phi=\rho+\bar{\rho}$. It is only in the absence of the interaction $V(\phi)$ that $\rho(\bar{\rho})$ would be chiral in the sense of being purely left (right) moving. But we will retain the definition in (2.3) of $\rho$ and $\bar{\rho}$ even for the non-conformal case $(V(\phi) \neq 0)$, and we will continue to call them chiral fields for simplicity. 
In the quantized theory, the fields $\phi$ and $\dot{\phi}$ obey the standard canonical equal-time commutation rules

$$
\begin{gathered}
{[\phi(x), \dot{\phi}(y)]_{t}=4 \pi i \delta(x-y),} \\
{[\phi(x), \phi(y)]_{t}=[\dot{\phi}(x), \dot{\phi}(y)]_{t}=0 .}
\end{gathered}
$$

Henceforth, all our operators, their products and commutators will be at some common time $t$. With this understanding, the symbol $t$ will not always be displayed. The canonical commutators in (2.4) form the basis of all our calculations. It must be remembered that they hold exactly regardless of whether the interaction $V(\phi)$ is present or not, or what form $V(\phi)$ has (as long as this interaction does not involve $\dot{\phi}$ ). We stress this obvious point to contrast our approach with certain conformal perturbation theoretical methods, which perturbatively employ expressions for time-ordered operator products that really hold only in the conformal $(V(\phi)=0)$ limit.

Given (2.4) the equal-time commutators of the chiral fields are

$$
\begin{gathered}
{[\rho(x), \rho(y)]=[\bar{\rho}(y), \bar{\rho}(x)]=-i \pi \epsilon(x-y),} \\
{[\rho(x), \bar{\rho}(y)]=-i \pi}
\end{gathered}
$$

Next, we separate fields into creation and annihilation parts for purposes of normal ordering, to render our operators finite in the ultraviolet. All our results arise basically due to short-distance singularities, but in this $(1+1)$ dimensional theory, care has also to be exercised regarding possible infrared divergences. Although our final results are in fact free of infrared divergences, we will keep careful track of infrared behavior. Let us normal order the fields $\phi$ and $\dot{\phi}$ (and thereby also $\rho$ and $\bar{\rho}$ through their definitions (2.3)) with respect to a free field of nonzero mass $m$. The resulting equal-time commutators at some common time $t$ between the creation $\left(\rho_{-}, \bar{\rho}_{-}\right)$and destruction $\left(\rho_{+}, \bar{\rho}_{+}\right)$parts are shown in Appendix A to be

$$
\begin{aligned}
{\left[\rho_{+}(x), \rho_{-}(y)\right] } & =K_{0}(m|x-y|)+\frac{1}{2} M(m|x-y|)-i \frac{\pi}{2} \epsilon(x-y) \\
{\left[\rho_{+}(x), \bar{\rho}_{-}(y)\right] } & =-\frac{1}{2} M(m|x-y|)-i \frac{\pi}{2}
\end{aligned}
$$




$$
\begin{aligned}
& {\left[\bar{\rho}_{+}(x), \bar{\rho}_{-}(y)\right]=K_{0}(m|x-y|)+\frac{1}{2} M(m|x-y|)+i \frac{\pi}{2} \epsilon(x-y)} \\
& {\left[\bar{\rho}_{+}(x), \rho_{-}(y)\right]=-\frac{1}{2} M(m|x-y|)+i \frac{\pi}{2}}
\end{aligned}
$$

where $K_{0}(z)$ is a Bessel function. As shown in Appendix A, the function $M(z)$ which obeys

$$
\frac{d^{2} M(z)}{d z^{2}}=-K_{0}(z)
$$

actually contains an infrared divergent, but $z$-independent integration constant. That will affect us only to the extent of having to insert an infrared renormalization constant into our definiton of vertex operators (see below). Aside from this, for the bulk of our work, we will need only the following equal-time commutators, obtained from eq (2.6) using $\phi=\rho+\bar{\rho}$,

$$
\begin{gathered}
{\left[\rho_{+}(x), \phi_{-}(y)\right]=K_{0}(m|x-y|)-i \pi \theta(x-y)=-\ln (i \bar{m}(x-y-i \epsilon))-\frac{i \pi}{2}+O\left((x-y)^{2}\right)} \\
{\left[\rho_{-}(x), \phi_{+}(y)\right]=-K_{0}(m|x-y|)-i \pi \theta(x-y)=\ln (-i \bar{m}(x-y+i \epsilon))-\frac{i \pi}{2}+O\left((x-y)^{2}\right)} \\
{\left[\bar{\rho}_{+}(x), \phi_{-}(y)\right]=K_{0}(m|x-y|)+i \pi \theta(x-y)=-\ln (-i \bar{m}(x-y+i \epsilon))+\frac{i \pi}{2}+O\left((x-y)^{2}\right)} \\
{\left[\bar{\rho}_{-}(x), \phi_{+}(y)\right]=-K_{0}(m|x-y|)+i \pi \theta(x-y)=\ln (i \bar{m}(x-y-i \epsilon))+\frac{i \pi}{2}+O\left((x-y)^{2}\right)}
\end{gathered}
$$

where $\epsilon \rightarrow 0$ represents our short-distance (ultraviolet) regularization. We see that these commutators are free of the infrared divergent function $M(z)$. The middle expression in each equation in (2.8) is exact, but we have also shown its behavior in the small (x-y) limit for later use. Here $\bar{m} \equiv \frac{1}{2} m e^{\gamma}$ where $\gamma$ is Euler's constant.

We will primarily be interested in vertex operators $W_{a, b}(x)$ defined by

$$
W_{a, b}(x) \equiv D_{a, b}: e^{i a \rho(x)+i b \bar{\rho}(x)}: \equiv D_{a, b} e^{i a \rho_{-}(x)+i b \bar{\rho}_{-}(x)} e^{i a \rho_{+}(x)+i b \rho_{+}(x)}
$$

where $D_{a, b}=\exp \left[-(a-b)^{2}(m-\mu) / 4 \mu\right]$ is a constant with the infrared cutoff $\mu \rightarrow 0$. This factor is inserted because of the infrared divergent constant in the commutators (2.6). Notice that $D_{a, b}$ is unity when $a=b$, i.e. when only the combination $\phi=\rho+\bar{\rho}$ is involved. The symbol :: refers to normal ordering with respect to a nonzero mass $m$ as outlined above. This $W_{a, b}$ is free of ultraviolet and infrared singularities. 
The regularized Hamiltonian of the quantized $S G$ theory can be written as $H=H_{0}+H_{I}$ where

$$
H_{0}=\frac{1}{4 \pi} \int d y\left(:\left(\frac{\partial \rho}{\partial y}\right)^{2}+\left(\frac{\partial \bar{\rho}}{\partial y}\right)^{2}:\right)
$$

and

$$
H_{I}=-\lambda_{m} \int: \cos \beta \phi(y): d y=-\frac{\lambda_{m}}{2} \int\left(W_{\beta, \beta}(y)+W_{-\beta,-\beta}(y)\right) d y
$$

Here $\lambda_{m}$ is the renormalized coupling constant. It is important to remember for our later use that $: \cos \beta \phi:$ is divergence-free, $\lambda_{m}$ has to be taken finite so that the energies and masses in the vacuum sector of the $S G$ model may be finite. The constant $\beta$ need not be renormalized and is taken to be in the range $0<\beta^{2}<2$ as required by Coleman ${ }^{5}$.

Two properties of $W_{a, b}$, obtainable from the commutators (2.5), (2.6) and (2.8), and useful for our calculations are

$$
\begin{aligned}
{\left[W_{a, b}(x),\left(\frac{\partial \rho}{\partial y}\right)^{2}\right.} & \left.+\left(\frac{\partial \bar{\rho}}{\partial y}\right)^{2}\right] \\
& =4 \pi i\left[:\left(a \frac{\partial \rho}{\partial x}-b \frac{\partial \bar{\rho}}{\partial x}\right) W_{a, b}: \delta(x-y)+\frac{a^{2}-b^{2}}{2} W_{a, b} \partial_{x} \delta(x-y)\right]
\end{aligned}
$$

and the equal-time operator product, obtained by using (2.8)

$$
\begin{aligned}
W_{a, b}(x) W_{c, c}(y) & =D_{a, b}: e^{i a \rho(x)+i b \bar{\rho}+i c \phi(y)}: \\
& \times \exp \left[-(a c+b c) K_{0}(m|x-y|)+i \pi(a c-b c) \theta(x-y)\right] .
\end{aligned}
$$

Note that the infrared factor $D_{a, b}$, which arises because this same factor is present in $W_{a, b}$ (see (2.9)), once again precisely offsets the infrared divergence of the vertex function on the r.h.s. The only singularity in (2.12) is at $\mathrm{x}=\mathrm{y}$ due to the $\theta$ function.

\section{VERTEX OPERATORS}

In this section we present some very interesting properties of vertex operators $W_{a, b}(x)$ in the SG theory. These properties turn out to be responsible, among other things for (a) the existence 
of an exactly conserved nonlocal current in the SG theory with terms only up to $O\left(\lambda_{m}\right)$ and (b) the absence of a "mass term" in the dynamics of the Mandelstam fermion operator even in the presence of the non-conformal $\left(-\lambda_{m}: \cos \beta \phi:\right)$ term in the Hamiltonian.

We will consider only those $W_{a, b}$ which satisfy

$$
(a-b) \beta=n
$$

where $n$ is an integer, positive, negative or zero. The reason for placing this restriction comes from the topological index of $W_{a, b}$, evaluated in ref. (2). As is well known, all finite energy configurations in the $S G$ theory must carry an integer value for the topological quantum number

$$
T \equiv \frac{\beta}{2 \pi} \int d x \frac{\partial \phi}{\partial x}=\frac{\beta}{2 \pi} \int d x\left(\frac{\partial \rho}{\partial x}+\frac{\partial \bar{\rho}}{\partial x}\right)
$$

From the commutation rule $(2.5)$, we see that

$$
\left[\frac{\partial \rho}{\partial x}+\frac{\partial \bar{\rho}}{\partial x}, W_{a, b}(y)\right]=\pi(a-b) \frac{\partial}{\partial x} \epsilon(x-y) W_{a, b}(y)
$$

Hence,

$$
\left[T, W_{a, b}(y)\right]=(a-b) \beta W_{a, b}(y)
$$

Thus the requirement (3.1) ensures that the $W_{a, b}$ have integer valued topological quantum numbers. Under the equivalence to the Thirring model, ${ }^{5}$ one expects the $W_{a, b}$ satisfying (3.1) to have a nonzero matrix element between the vacuum sector and the $n$-fermion sector.

Take any such $W_{a, b}(x)$ satisfying $(a-b) \beta=n$, where to be specific let $n>0$. The $n \leq 0$ cases are treated similarly (see below). Consider the commutator of such a $W_{a, b}$ with the interaction Hamiltonian $H_{I}=-\frac{\lambda_{m}}{2} \int d y\left(W_{\beta, \beta}(y)+W_{-\beta,-\beta}(y)\right)$. By translation invariance it is sufficient to consider $W_{a, b}(x)$ at $x=0$.

We have, using (2.12),

$$
\begin{gathered}
{\left[W_{a, b}(0), W_{\beta, \beta}(y)\right]=C_{+}(y)\left\{\left(\exp \left[-(a+b) \beta K_{0}(m|-y|)+i \pi(a-b) \beta \theta(-y)\right]\right.\right.} \\
\left.-\exp \left[-(a+b) \beta K_{0}(m|y|)+i \pi(a-b) \beta \theta(y)\right]\right\}
\end{gathered}
$$

where 


$$
C_{ \pm}(y) \equiv D_{a, b}: e^{i a \rho(0)+i b \bar{\rho}(0) \pm i \beta \phi(y)}:
$$

are nonsingular operators.

We see that since $(a-b) \beta=n$, the r.h.s. of (3.5) vanishes for $y \neq 0$. Hence, if this commutator were to be integrated over $y$, the only contribution will come from possible singularities at $y=0$. Therefore, we can use the short distance behavior of these commutators, given in (2.8) to write

$$
\begin{aligned}
{\left[W_{a, b}(0),-\frac{\lambda_{m}}{2} \int d y W_{\beta, \beta}(y)\right] } & \\
=-\frac{\lambda_{m}}{2} \lim _{\epsilon \rightarrow 0} \int d y C_{+}(y) \quad & \left\{(\bar{m}(-y-i \epsilon))^{a \beta}(\bar{m}(-y+i \epsilon))^{b \beta} e^{i \pi(a-b) \beta}\right. \\
\left.-(\bar{m}(-y+i \epsilon))^{a \beta}(\bar{m}(-y-i \epsilon))^{b \beta} e^{-i \pi(a-b) \beta}\right\} & \\
=-\frac{\lambda_{m}}{2}(\bar{m})^{(a+b) \beta} \lim _{\epsilon \rightarrow 0} \int d y & C_{+}(y)\left(y^{2}+\epsilon^{2}\right)^{b \beta}\left\{(y+i \epsilon)^{n}-(y-i \epsilon)^{n}\right\}
\end{aligned}
$$

Similarly

$$
\begin{aligned}
& {\left[W_{a, b}(0),-\frac{\lambda_{m}}{2} \int d y W_{-\beta,-\beta}(y)\right]} \\
& \quad=-\frac{\lambda_{m}}{2}(\bar{m})^{-(a+b) \beta} \lim _{\epsilon \rightarrow 0} \int d y C_{-}(y)\left(y^{2}+\epsilon^{2}\right)^{-a \beta}\left\{(y+i \epsilon)^{n}-(y-i \epsilon)^{n}\right\} .
\end{aligned}
$$

The operators $C_{ \pm}(y)$ defined in (3.6) are properly regularized and singularity-free. The integrands in (3.7-3.8) vanish as $\epsilon \rightarrow 0$ for any $y \neq 0$. Hence, the $y$-integrals will vanish unless there is a sufficiently strong singularity at $y=0$. For any given positive integer $n$, such singularities in the commutator can arise only for sufficiently negative $b$ in (3.7) or positive $a$ in (3.8). Very roughly, one would estimate that the commutator (3.7) behaves like $\left(y^{2}+\epsilon^{2}\right)^{(b \beta+n)} C_{+}(y) \partial_{y}^{n-1} \delta(y)$ and that its integral over $y$ would behave as $\epsilon^{2 \beta b+n+1}$ with $\epsilon \rightarrow 0$. This expectation is made more precise in Appendix B where we evaluate, for any positive integer $n$, any real $p$ and any nonsingular function $C(y)$, the integral

$$
I \equiv \lim _{\epsilon \rightarrow 0} \int d y\left(y^{2}+\epsilon^{2}\right)^{p}\left((y+i \epsilon)^{n}-(y-i \epsilon)^{n}\right) C(y)
$$

It is shown here that this integral vanishes if $2 p+n+1>0$ when $n$ is odd and if $2 p+n+2>0$ when $n$ is even. Let us apply this to eq. (3.7) and (3.8). The term in (3.7) vanishes if $2 p \beta+n+1>0$ for $n$ odd and $2 b \beta+n+2>0$ for $n$ even. Along with (3.1), this condition becomes 


$$
a \beta>\frac{n-1}{2} \quad \text { for } n \text { odd }
$$

and

$$
a \beta>\frac{n-2}{2} \quad \text { for } n \text { even } .
$$

Similarly, (3.8) vanishes if $-2 a \beta+n+1>0$ for $n$ odd and $-2 a \beta+n+2>0$ for $n$ even, which amounts to

$$
a \beta<\frac{n+1}{2} \quad \text { for } n \text { odd }
$$

and

$$
a \beta<\frac{n+2}{2} \quad \text { for } n \text { even } .
$$

By adding (3.7) and (3.8), we therefore see (since the renormalized coupling $\lambda_{m}$ is finite) that

$$
\left[W_{a, b}(0), H_{I}\right]=\left[W_{a, b}(0),-\lambda_{m} \int d y: \cos \beta \phi(y):\right]=0
$$

for any finite $\lambda_{m}$, provided

$$
\frac{n-1}{2}<a \beta<\frac{n+1}{2} \quad \text { for } n \text { odd }
$$

or

$$
\frac{n-2}{2}<a \beta<\frac{n+2}{2} \quad \text { for } n \text { even }
$$

where we recall that $b$ is fixed for any given $a$ and $n$ by (3.1).

We have analyzed the case when the integer $n>0$. Similar results also hold for $n \leq 0$. When $n=0, a=b$ and $W_{a, b}=: e^{i a \phi}:$ and clearly this commutes with $: \cos \beta \phi(y):$ for all $a$. The result for the $n<0$ case is treated in the same way as the $n>0$ case, with the roles of $a$ and $b$ interchanged. The condition (3.13) continues to hold unchanged even for $n<0$. Hence, for any integer $n, W_{a, b}$ with $a$ and $b$ satisfying (3.1) and (3.13) commutes with the interaction Hamiltonian $H_{I}$.

It should be emphasized that these results cannot be circumvented by a further ultraviolet renormalization of $\lambda_{m}$ as $\epsilon \rightarrow 0$. The operator : $\cos \beta \phi:$ has been rendered finite in the vacuum 
sector by normal ordering. Hence $\lambda_{m}$ has to be taken finite in order to get $a$ finite energies into the vacuum sector. There is no further freedom to infinitely renormalize $\lambda_{m}$ to compensate for the zeroes of (3.7) and (3.8) as $\epsilon \rightarrow 0$.

The vanishing of (3.12) holds when the inequalities (3.13) are satisfied along with (3.1). A marginal situation arises when these conditions become equalities. For such cases, we apply equations (B.5b) and (B.6b) in Appendix B to the integrals in (3.7-3.8). We see that when

$$
(a-b) \beta=n, \quad a \beta=\frac{n \pm 1}{2} \quad(\text { odd } n)
$$

or

$$
(a-b) \beta=n, \quad a \beta=\frac{n \pm 2}{2} \quad(\text { even } n)
$$

then the commutator $\left[W_{a, b}(x), H_{I}\right]$ is finite and generically nonzero, as $\epsilon \rightarrow 0$. When the values of (a,b) fall outside the above ranges, i.e. when $a \beta>\frac{n+1}{2}$, or $a \beta<\frac{n-1}{2}$ (for $n$ odd) and $\alpha \beta>\frac{n+2}{2}$ or $\alpha \beta<\frac{n-2}{2}$ (for $n$ even), this commutator diverges.

The vanishing of the commutator (3.12) for some ranges of $a$ and $b$ has several interesting consequences. One application arises in the derivation of exact nonlocal currents for the SG model $^{2,4}$. Since the derivation of these currents using canonical equal-time methods has already been given briefly in ref. (4) and in Appendix A, we will not go into all the details here, other than to point out how our result (3.12) is crucial for the success of the Zamoldchikov's argument ${ }^{1}$ (3.12) involved there. This argument says that in such currents obtained for off-conformal theories with relevant perturbations (of which the SG model is an example), terms of second and higher order in the perturbation will be strictly absent. In the papers of Zamoldchikov ${ }^{1}$ and Bernard and Leclair, ${ }^{2}$ this argument is based on scale-weight counting within the conformal perturbation theoretic framework. The corresponding manifestation of this phenomenon in canonical equal-time quantization relies on our (3.12). To see this, recall ${ }^{2,4}$ that to obtain this nonlocal current in the SG theory, one starts with the operator $W_{\frac{2}{\beta}, 0}=D_{\frac{2}{\beta}}, 0: e^{\frac{2 i}{\beta} \rho(x, t)}:$. In the absence of the interaction $H_{I}=-\lambda_{m}: \cos \beta \phi:, \rho(x, t)$ is purely left moving, so that, to order $\left(\lambda_{m}\right)^{0}$,

$$
\partial_{-} W_{\frac{2}{\beta}, 0}(x t) \equiv\left(\partial_{t}-\partial_{x}\right) W_{\frac{2}{\beta}, 0}(x, t)=0
$$


When $H_{I}$ is turned on, eq. (3.15) is modified to

$$
\partial_{-} W_{\frac{2}{\beta}, 0}=\left[W_{\frac{2}{\beta}, 0}(x, t), H_{I}\right]
$$

Notice that $W_{\frac{2}{\beta}, 0}$ corresponds to $a=\frac{2}{\beta}, b=0$ and $n=\beta(a-b)=2$. Thus it satisfies (3.1) and is a legitimate operator with topological number 2. Further, since it satisfies the equality condition (3.14b), its commutator with $H_{I}$ will be finite and nonzero. Indeed this is what the explicit calculation in ref. (4) shows, where after some algebra, the eq. (3.16) is put in the form (see eq. (3.8) and (3.9) of ref. 4),

$$
\partial_{-} W_{\frac{2}{\beta}, 0}(x)=\left(B_{1} \partial_{+}+B_{2} \partial_{-}\right) W_{\frac{2}{\beta}-\beta,-\beta}(x)+B_{3}\left[W_{\frac{2}{\beta}-\beta,-\beta}(x), H_{I}\right]
$$

where $B_{1}, B_{2}$ and $B_{3}$ are constants of order $\lambda_{m}$. Now, the last term in (3.17) is $O\left(\lambda_{m}\right)$ since $B_{3}$ and $H_{I}$ are each of order $\lambda_{m}$. However, consider the operator $W_{\frac{2}{\beta}-\beta,-\beta}$. It corresponds to $a=\frac{2}{\beta}-\beta$ and $b=-\beta$, so that $(a-b) \beta=2$, once again corresponding to topological number 2 . But it obeys the inequality $(3.13 \mathrm{~b})$, since

$$
\frac{n-2}{2}(=0)<a \beta\left(=2-\beta^{2}\right)<\frac{n+2}{2}(=2)
$$

for all $0<\beta^{2}<2$ which, according to Coleman $^{5}$, is the full range of $\beta$ for which the SG theory exists. Consequently, the commutator in the last term of (3.17) vanishes. The remaining terms yield an exactly conserved current containing only terms up to $O\left(\lambda_{m}\right)$, and the Zamoldchikov conjucture holds in this canonical quantization framework as well. For more properties of these currents, see ref. (2, 3 and 4), as well as later sections of this paper.

\section{DYNAMICS OF THE MANDELSTAM OPERATOR}

Another important consequence of the vanishing of the commutator $\left[W_{a, b}(x), H_{I}\right]$ for a range of $a$ and $b$ is that the time evolution of $W_{a, b}(x)$ is not affected by the presence of $H_{I}$. These $W_{a, b}$ obey a scale invariant field equation. A particularly interesting example of such $W_{a, b}$ is the Mandelstam operator for the bosonic representation of the Fermi field. 
Consider the time evolution of the operator $W_{a, b}(x, t)$ with $a$ and $b$ satisfying (3.1) and (3.13). We have, in the Heisenberg representation,

$$
\frac{\partial}{\partial t} W_{a, b}(x, t)=-i\left[W_{a, b}, H_{0}+H_{I}\right]
$$

where the Hamiltonian is given in (2.10). Since this $W_{a, b}(x)$ commutes with the interaction Hamiltonian, its time evolution is determined solely by the kinetic term in $H_{0}$. We have, using (2.10), (2.11) and (3.12),

$$
\frac{\partial}{\partial t} W_{a, b}(x, t)=-i\left[W_{a, b}(x), \frac{1}{4 \pi} \int d y:\left(\left(\frac{\partial \rho}{\partial y}\right)^{2}+\left(\frac{\partial \bar{\rho}}{\partial y}\right)^{2}\right):\right] .
$$

We can use the commutators in (2.11) to evaluate this as

$$
\frac{\partial}{\partial t} W_{a, b}(x, t)=i:\left(a \frac{\partial \rho}{\partial x}-b \frac{\partial \bar{\rho}}{\partial x}\right) W_{a, b}(x, t):
$$

This is a very interesting result. The SG theory is not a scale invariant theory because of the interaction term $-\lambda_{m}: \cos \beta \phi:$. Nevertheless, those vertex functions $W_{a, b}(x)$ which satisfy (3.13) and (3.1), obey an equation of motion (3.17) which is scale invariant under $x \rightarrow k x, t \rightarrow k t$. (Recall that $\frac{\partial \rho}{\partial x}$ and $\frac{\partial \bar{\rho}}{\partial x}$ have scale dimension of unity; see sec. 4). This is because these $W_{a, b}$ commute with the scale-breaking SG interaction.

An especially inetresting example of this is the well-known Mendelstam construction ${ }^{6}$ of the bosonic representation for the Fermi field developed for relating the SG model to the massive Thirring model (MTM). Recall that it has the form (eq. 2.8 of ref. 6)

$$
\Psi=\left(\begin{array}{l}
\psi_{1} \\
\psi_{2}
\end{array}\right)
$$

with

$$
\begin{gathered}
\psi_{1}=N: \exp \left[-\frac{i}{2}\left(\frac{1}{\beta}+\beta\right) \rho+\frac{i}{2}\left(\frac{1}{\beta}-\beta\right) \bar{\rho}\right] \\
\psi_{2}=-i N: \exp \left[-\frac{i}{2}\left(\frac{1}{\beta}-\beta\right) \rho+\frac{i}{2}\left(\frac{1}{\beta}+\beta\right) \bar{\rho}\right]
\end{gathered}
$$

where $N$ is a constant. (In converting our notation to that of Mandelstam ${ }^{6}$ and Coleman ${ }^{5}$, change $\beta \rightarrow \beta / \sqrt{4 \pi}$ and $\phi \rightarrow \sqrt{4 \pi} \phi)$. Thus, both $\psi_{1}$ and $\psi_{2}$ belong to the class of $W_{a, b}$ operators, with 


$$
a_{1}=-\frac{1}{2}\left(\frac{1}{\beta}+\beta\right), b_{1}=\frac{1}{2}\left(\frac{1}{\beta}-\beta\right),\left(a_{1}-b_{1}\right) \beta=n_{1}=-1
$$

and

$$
a_{2}=-\frac{1}{2}\left(\frac{1}{\beta}-\beta\right), b_{2}=\frac{1}{2}\left(\frac{1}{\beta}+\beta\right),\left(a_{2}-b_{2}\right) \beta=n_{2}=-1
$$

The condition for the vanishing of $\left[\psi_{1}(x), H_{I}\right]$ and $\left[\psi_{2}(x), H_{I}\right]$, transcribed from (3.13a), reduces respectively to

$$
-1<a_{1} \beta=-\frac{1}{2}\left(1+\beta^{2}\right)<0
$$

and

$$
-1<a_{2} \beta=-\frac{1}{2}\left(1-\beta^{2}\right)<0
$$

Both these conditions are satisfied in the regime $0<\beta^{2}<1$.

Therefore, for $\beta^{2}<1$, the time evolution of the operators $\psi_{1}$ and $\psi_{2}$ is governed solely by the unperturbed Hamiltonian $H_{0}$ in (2.10a). We have, as a special case of (4.3),

$$
\frac{\partial \psi_{1}}{\partial t}=i:\left(\frac{1}{2}\left(-\beta-\frac{1}{\beta}\right) \frac{\partial \rho}{\partial x}-\frac{1}{2}\left(\frac{1}{\beta}-\beta\right) \frac{\partial \bar{\rho}}{\partial x}\right) \psi_{1}:
$$

and

$$
\frac{\partial \psi_{2}}{\partial t}=i:\left(\frac{1}{2}\left(\beta-\frac{1}{\beta}\right) \frac{\partial \rho}{\partial x}-\frac{1}{2}\left(\beta+\frac{1}{\beta}\right) \frac{\partial \bar{\rho}}{\partial x}\right) \psi_{2}: .
$$

The "mass term" expected in these equations is absent even though the SG Hamiltonian contains the interaction $H_{I}=-\lambda_{m} \cos \beta \phi:$ which is the analogue of the appropriately regularized mass term $m_{F} Z \bar{\Psi} \Psi$ in the massive Thirring model ${ }^{5,6}$. To put it another way the contribution of $H_{I}$ to $\frac{\partial \psi_{1}}{\partial t}$ in the $S G$ theory behaves (upon inserting the values for $a$ and $b$ from (4.5) into (3.7-3.8)) as

$$
\left[\psi_{1,2}, H_{I}\right]=\lim _{\epsilon \rightarrow 0}(\bar{m} \epsilon)^{\left(1-\beta^{2}\right)}\left(\frac{\lambda_{m}}{2 \bar{m}}\right) \psi_{2,1}
$$

This has the correct dimensions and structure of what the "mass term" should have, but it vanishes as $\epsilon \rightarrow 0$ when $\beta^{2}<1$. We repeat again that the SG coupling constant $\lambda_{m}$ multiplying the normal ordered : $\cos \beta \phi$ : has to be taken finite, to keep energies finite in the SG vacuum sector. There 
is no freedom to further renormalize $\lambda_{m}$ to offset the vanishing of (4.9) as $\epsilon \rightarrow 0$. Also since (4.9) is linear in $\psi_{1,2}$, no $\epsilon$-dependent rescaling of $\psi_{1,2}$ will restore this mass term. Lastly the constant $\bar{m}=\frac{1}{2} m e^{\gamma}$ is finite and nonzero, where $m$ is our normal ordering mass. Thus, we see no way to circumvent the vanishing of this "mass" term.

Therefore, in the range $\beta^{2}<1$ (which corresponds ${ }^{5}$ to the Thirring coupling $g>0$ ), the Mandelstam Fermi field does not develop a mass term, and instead obeys the scale invariant equation of motion (4.7-4.8) that it would have in the massless Thirring model. Since the vector current of the Thirring model corresponds under bosonization rules to $\beta \epsilon^{\mu \nu} \partial_{\nu} \phi$, it can be checked that eqs.(4.7-4.8) indeed correspond to the massless Thirring equation.

When $\beta^{2}=1($ i.e. $g=0)$, one has an example of the marginal situation described in eq. (3.14). Then the commutator $\left[\psi_{1,2}(x), H_{I}\right]$ is finite and a nonzero mass term appears in $\partial_{t} \psi_{1,2}$. This is also evident from eq (4.4). Thus, it is only when $\beta^{2}=1$, which corresponds to free massive fermions with zero Thirring coupling, that the Mandelstam construction $\Psi[\phi]$ has the same dynamics in the SG model as the Fermi field does in the corresponding Dirac Theory. When $\beta^{2}<1$, the desired mass term does not develop, and when $\beta^{2}>1$, the commutator $\left[\psi_{1,2}, H_{I}\right]$ diverges. Other problems with the Mandelstam operator when $\beta^{2} \neq 1$ had been noted by Schroer and Truong long ago ${ }^{7}$ Our results dealing with its time-evolution are possible related to those.

It should be pointed out that our derivations give no disagreement with the bosonization rules developed by Coleman ${ }^{5}$ which perturbatively relate correlation functions of fermion bilinears : $\bar{\Psi} \Psi$ : and $\bar{\Psi} \gamma^{\mu} \Psi$ in the Thirring model to those of : $\cos \beta \phi:$ and $\beta \epsilon^{\mu \nu} \partial_{\nu} \phi$ respectively in the massless boson theory. Nor do we disagree with any equation explicitly given in the Mandelstam paper $^{6}$. In that paper (see eq. 4.13 of ref. 6) this mass term is not actually evaluated. It is left in the form of a commutator of the form $\left[\psi, M \int Z \bar{\psi} \psi\right]$. Our point is that this commutator vanishes when explicitly evaluated in the SG system for $\beta^{2}<1(g>0)$, and survives as a finite term only when $\beta^{2}=1(g=0)$. To that extent, the Mandelstam operator does not reproduce in the SG model the same time-evolution as the Fermi field in the MTM, except when $\beta^{2}=1$. For $\beta^{2}<1$, the Mandelstam operator obeys a scale-invariant massless field equation even though the SG Hamiltonian carries the scale-breaking $\lambda_{m}: \cos \beta \phi$ : interaction - a somewhat surprising 
result.

Finally, note that while we have highlighted the case of the Mandelstam operator $\psi$ because of its obvious importance, that is just one example of similar behavior by a whole family of vertex operators $W_{a, b}$ satisfying the conditions (3.1) and (3.13). For arbitrary $n$, they are generalizations of the Mandelstam construction corresponding to arbitrary fermion number. When $a$ satisfies (3.13), they all satisfy scale-invariant field equations.

\section{TRANSFORMATION PROPERTIES OF $W_{a, b}$ AND CURRENTS}

In this section, we shall work out the transformation properties of the vertex operator $W_{a, b}(x, t)$ and currents $J_{ \pm}$under Lorentz and scale transformations. We assume that all operators are normalordered with respect to the reference mass $m$. We shall omit its subscript $m$ and denote the coupling constant simply as $\lambda$. In Appendix A, we show how $W_{a, b}$ of different reference masses are related. It is straightforward to verify that the transformation laws established in this section under the Lorentz and the scale transformations are valid for all reference masses.

(a) Lorentz Transformation

From the Lagrange function

$$
\mathcal{L}=\frac{1}{8 \pi} \partial_{\mu} \phi \partial^{\mu} \phi-V(\phi)
$$

we can construct the stress tensor as

$$
T_{\mu \nu}=\frac{1}{4 \pi} \partial_{\mu} \phi \partial_{\nu} \phi-g_{\mu \nu} \mathcal{L}
$$

We assume that $\mathcal{L}, T_{\mu \nu}$ and $M$ to be introduced are all normal- ordered w.r.t. mass $m$. From the definition of $\rho$ and $\bar{\rho}$ in $(2.3)$, we have

$$
\begin{aligned}
& \phi=\rho+\bar{\rho} \\
& \dot{\phi}=\frac{\partial \rho}{\partial x}-\frac{\partial \bar{\rho}}{\partial x} .
\end{aligned}
$$

The Lorentz boost generator is 


$$
\begin{aligned}
M & =\int d y\left(y_{0} T_{01}-y_{1} T_{00}\right) \\
& =\int d y\left[t \frac{1}{4 \pi} \dot{\phi} \frac{\partial \phi}{\partial y}+y\left(\frac{1}{8 \pi} \dot{\phi}^{2}+\frac{1}{8 \pi}\left(\frac{\partial \phi}{\partial y}\right)^{2}+V(\phi)\right)\right] \\
& =\frac{1}{4 \pi} \int d y\left[(y+t)\left(\frac{\partial \rho}{\partial y}\right)^{2}+(y-t)\left(\frac{\partial \bar{\rho}}{\partial y}\right)^{2}+4 \pi y V(\rho+\bar{\rho})\right] .
\end{aligned}
$$

It is straightforward to verify that $\phi, \frac{\partial \rho}{\partial x}$ and $\frac{\partial \bar{\rho}}{\partial x}$ all transform covariantly under $M$ with well-defined Lorentz weights $0, \pm 1$. However, variables $\rho$ and $\bar{\rho}$ transform noncovariantly as

$$
\begin{aligned}
& {[\rho(x, t), M]=i\left(t \partial_{x}+x \partial_{t}\right) \rho(x, t)-\int d y(x-y)[\rho(x, t), V(\phi(y, t))]} \\
& {[\bar{\rho}(x, t), M]=i\left(t \partial_{x}+x \partial_{t}\right) \bar{\rho}(x, t)-\int d y(x-y)[\bar{\rho}(x, t), V(\phi(y, t))] .}
\end{aligned}
$$

The last potential-dependent terms in (5.6) arise from the nonlocal definition of $\rho$ and $\bar{\rho}(2.3)$ involving an integration over space at a fixed time. Under a Lorentz transformation, the equaltime frame is changed. This change induces the nonlocal potential-dependent terms in (5.6).

The vertex operator $W_{a, b}(x) \equiv D_{a, b}: e^{i a \rho(x)+i b \bar{\rho}(x)}:$ defined in (2.9) w.r.t a finite mass $m$ transforms under $M$ as

$$
\left[W_{a, b}(x), M\right]=i\left[t \partial_{x}+x \partial_{t}+\frac{1}{2}\left(a^{2}-b^{2}\right)\right] W_{a, b}(x)-\int d y(x-y)\left[W_{a, b}(x), V(\phi(y))\right]
$$

where $\frac{1}{2}\left(a^{2}-b^{2}\right)$ represents the Lorentz weight in the absence of $V(\phi)$.

To verify (5.7), we can follow the same method as described in ref. 4. The change of reference mass only leads to an additive constant in $M$, and does not affect the commutator.

The existence of the extra potential-dependent term in (5.7) implies that the vertex operator $W_{a, b}(x)$ in general does not transform covariantly with a well-defined Lorentz weight. However, it should be noted that for the special class of vertex operators $W_{a, b}(x)$ and their adjoint $W_{-a,-b}(x)$ studied in section 3 , defined by

$$
\beta(a-b)=n>0, \quad n \text { an integer }
$$

and

$$
\frac{n+1}{2}>a \beta>\frac{n-1}{2}, \quad n=\text { odd }
$$




$$
\frac{n}{2}+1>a \beta>\frac{n}{2}-1, \quad n=\text { even }
$$

the last commutator terms in (5.7) vanish identically. The cancellation follows the same argument described in sec. 3. The extra $(x-y)$ factor only makes the integration vanish faster. These special vertex operators not only satisfy simple equations of motion as shown in sec. 3 , they also transform covariantly with Lorentz weight $\frac{1}{2}\left(a^{2}-b^{2}\right)$.

\section{(b) Scale Transformation}

The generator of the scale transformation is

$$
\begin{aligned}
S & =\int d x x^{\mu} T_{0 \mu}=\int d x\left(t T_{00}+x T_{01}\right) \\
& =\frac{1}{4 \pi} \int d y\left[(y+t)\left(\frac{\partial \rho}{\partial y}\right)^{2}-(y-t)\left(\frac{\partial \bar{\rho}}{\partial y}\right)^{2}+4 \pi t V(\rho+\bar{\rho})\right] .
\end{aligned}
$$

Unlike the Lorentz generator, $S$ is not a constant of motion. The time derivative of $S$ equals to the space integral of $T_{\mu}^{\mu}$, giving

$$
\frac{d S}{d t}=\int d x\left(T_{\mu}^{\mu}\right)=2 \int d y V(\phi(y))
$$

In spite of its time dependence, we find it useful to classify field variables according to their transformation properties under the scale transformation $S$. This is analog to describe a system under a nonspherical disturbance by its angular momentum states. From (5.11), we can work out the transformation properties of $\rho$ and $\bar{\rho}$ under $S$ as

$$
\begin{aligned}
& {[\rho(x, t), S(t)]=i\left(t \frac{\partial}{\partial t}+x \frac{\partial}{\partial x}\right) \rho(x, t)} \\
& {[\bar{\rho}(x, t), S(t)]=i\left(t \frac{\partial}{\partial t}+x \frac{\partial}{\partial x}\right) \bar{\rho}(x, t) .}
\end{aligned}
$$

Thus, $\rho$ and $\bar{\rho}$ transform covariantly with zero scaling weight.

We can work out the transformation properties of the vertex operator $W_{a, b}(x, t)$ in a way similar to those presented in subsection (a). They are

$$
\left[W_{a, b}(x, t), S(t)\right]=i\left[t \frac{\partial}{\partial t}+x \frac{\partial}{\partial x}+\frac{1}{2}\left(a^{2}+b^{2}\right)\right] W_{a, b}(x, t)
$$


Thus $W_{a, b}(x, t)$ transforms covariantly under $S(t)$ with a scaling weight $\frac{1}{2}\left(a^{2}+b^{2}\right)$ for all $a$ and $b$, unlike the case of Lorentz spin. This is a bit surprising since this non-conformal theory is Lorentz invariant, but violates scale invariance. Of course the scale generator $S(t)$ is not conserved in time.

\section{(c) Transformations of Nonlocal Currents}

In this subsection, we shall work out the transformation laws of the currents $J_{ \pm}$derived in ref. 4, under Lorentz and scale transformations. To examine the Lorentz covariance of the currents, we start with

$$
\left[A_{\frac{2}{\beta}}(x), M\right]=i\left(t \partial_{x}+x \partial_{t}+\frac{2}{\beta^{2}}\right) A_{\frac{2}{\beta}}(x)+\int d y(x-y)\left[A_{\frac{2}{\beta}}(x), \lambda \cos (\beta \phi(y))\right]
$$

Note that $A_{\frac{2}{\beta}} \equiv W_{\frac{2}{\beta}}, 0$ corresponds to the special class of $W_{a, b}$ with $a=\frac{2}{\beta}, b=0, \beta(a-b)=2$, but is on the boundary of the region $2 \geq \frac{2}{\beta} \geq 0$. For this case as shown in sec. 3 the commutator $\left[A_{\frac{2}{\beta}}(x), \cos \beta \phi(y)\right]$ does not vanish, but can be evaluated as

$$
\begin{aligned}
\lambda \int & d y(x-y)\left[A_{\frac{2}{\beta}}(x), \cos \beta \phi(y)\right] \\
& =\frac{\lambda}{2 \bar{m}^{2}} \int d y(x-y)\left(-2 \pi i \partial_{x} \delta(x-y)\right) W_{\frac{2}{\beta}-\beta,-\beta}(y) \\
& =\frac{i \lambda \pi}{\bar{m}^{2}} W_{\frac{2}{\beta}-\beta,-\beta}(x) .
\end{aligned}
$$

Hence,

$$
\left[A_{\frac{2}{\beta}}, M\right]=i\left(t \partial_{x}+x \partial_{t}+\frac{2}{\beta^{2}}\right) A_{\frac{2}{\beta}}+\frac{i \lambda \pi}{\bar{m}^{2}} W_{\frac{2}{\beta}-\beta,-\beta}
$$

Similarly, we have

$$
\begin{aligned}
{\left[W_{\frac{2}{\beta}-\beta,-\beta}(x), M\right]=} & i\left(t \partial_{x}+x \partial_{t}+\frac{2}{\beta^{2}}-2\right) W_{\frac{2}{\beta}-\beta,-\beta}(x) \\
& -\int d y(x-y)\left[W_{\frac{2}{\beta}-\beta,-\beta}(x), \lambda \cos \beta \phi(y)\right]
\end{aligned}
$$

For $0<\beta^{2}<2, W_{\frac{2}{\beta}-\beta,-\beta}$ belongs to the special class of vertex functions described in (5.10), and the commutator vanishes. We have

$$
\left[W_{\frac{2}{\beta}-\beta}, M\right]=i\left(t \partial_{x}+x \partial_{t}+\frac{2}{\beta}-2\right) W_{\frac{2}{\beta}-\beta,-\beta}
$$


Eqs. (5.17) and (5.19) imply that $J_{ \pm}$defined in Appendix A transform covariantly

$$
\begin{gathered}
{\left[J_{+}, M\right]=i\left(t \partial_{x}+x \partial_{t}+\frac{2}{\beta^{2}}\right) J_{+}} \\
{\left[J_{-}, M\right]=i\left(t \partial_{x}+x \partial_{t}+\frac{2}{\beta^{2}}-2\right) J_{-} .}
\end{gathered}
$$

Notice that the Lorentz weights of $J_{+}$and $J_{-}$differ by 2 . Hence the conservation equation $\partial_{-} J_{+}+$ $\partial_{+} J_{-}=0$ will also be Lorentz covariant.

The transformation laws of $A_{\frac{2}{\beta}}$ and $W_{\frac{2}{\beta}-\beta,-\beta}$ under S can be computed easily as

$$
\begin{gathered}
{\left[A_{\frac{2}{\beta}}(x, t), S(t)\right]=i\left(t \frac{\partial}{\partial t}+x \frac{\partial}{\partial x}+\frac{2}{\beta^{2}}\right) A_{\frac{2}{\beta}}(x, t)} \\
{\left[W_{\frac{2}{\beta}-\beta,-\beta}(x, t), S(t)\right]=i\left(t \frac{\partial}{\partial t}+x \frac{\partial}{\partial x}+\frac{2}{\beta^{2}}-2+\beta^{2}\right) W_{\frac{2}{\beta}-\beta,-\beta} .}
\end{gathered}
$$

Since these two terms have different scaling weights, as their linear combination $J_{+}$does not have a well-defined scale covariant weight, although $J_{-}$does. The conservation equation $\partial_{-} J_{+}+\partial_{+} J_{-}=0$ also is not scale-covariant.

\section{ACKNOWLEDGMENTS}

R. Rajaraman thanks the Physics Department, University of Illinois at Urbana- Champaign for their hospitality. This work was supported in part by the U.S. National Science Foundation under grant No. 9200148 .

\section{APPENDIX A: RENORMALIZATION MASS AND INFRARED CUTOFF}

For calculations in the SG theory, we often encounter both the infrared and the ultraviolet divergences. To achieve ultraviolet finite results, we introduce operators in normal-ordered forms, expressed in terms of the creation and annihilation operators of a free field of mass $m$. We refer to this mass $m$ as the normal-ordering reference mass. In terms of these normal-ordered operators, the ultraviolet divergences often cancel out. In ref. 4, we choose the reference mass to be zero for 
calculational convenience. However, this convenience comes with a price. Most of the calculations with zero reference mass have infrared divergences. To achieve finite results, it is necessary to introduce infrared momentum cutoff $\mu$. On the other hand, the calculation with a finite reference mass is more complicated. One of the purposes of this appendix is to relate operators defined with respect to different reference masses, including zero reference mass. The transformation properties among general vertex functions involve subtleties which do not appear to be well known. We shall also work out explicitly current conservation laws completely in the framework of a finite reference mass.

The interaction term in the SG theory is

$$
V(\phi)=-\lambda_{0}(: \cos \beta \phi:)_{0}=-\lambda_{m}(: \cos \beta \phi:)_{m}
$$

where subscripts 0 and $m$ indicate the normal-ordering reference masses. The normal-ordered operators and the coupling constants are related by

$$
\begin{aligned}
(: \cos \beta \phi:)_{m} & =\left(\frac{2 \mu}{\mu+\sqrt{m^{2}+\mu^{2}}}\right)^{\beta^{2}}(: \cos \beta \phi:)_{0} \\
\lambda_{m} & =\left(\frac{\mu+\sqrt{m^{2}+\mu^{2}}}{2 \mu}\right)^{\beta^{2}} \lambda_{0} .
\end{aligned}
$$

For operators and coupling constants associated with two different masses $m, m^{\prime}>>\mu$, we have

$$
\begin{aligned}
(: \cos \beta \phi:)_{m^{\prime}} & =\left(\frac{m}{m^{\prime}}\right)^{\beta^{2}}(: \cos \beta \phi:)_{m} \\
\lambda_{m}^{\prime} & =\left(\frac{m^{\prime}}{m}\right)^{\beta^{2}} \lambda_{m} .
\end{aligned}
$$

Eqs. (A.4) and (A.5) contain neither ultraviolet nor infrared cutoff dependence.

Turning to general vertex functions $\exp (i a \rho(x)+i b \bar{\rho}(x))$, we define the normal ordered operator as

$$
(: \exp (i a \rho(x)+i b \bar{\rho}(x)):)_{m}=e^{i a \rho_{-}+i b \bar{\rho}_{-}} e^{i a \rho_{+}+i b \bar{\rho}_{+}}
$$

where $\rho_{ \pm}$and $\bar{\rho}_{ \pm}$are related to creation and annihilation operators by 


$$
\begin{gathered}
\rho_{+}(x, t)=\int \frac{d k}{\sqrt{\omega}} a(k, t) \frac{1}{2}\left(1-\frac{\omega}{k}\right) e^{i k x} \\
\rho_{-}(x, t)=\int \frac{d k}{\sqrt{\omega}} a^{+}(k, t) \frac{1}{2}\left(1-\frac{\omega}{k}\right) e^{-i k x} \\
\bar{\rho}_{+}(x, t)=\int \frac{d k}{\sqrt{\omega}} a(k, t) \frac{1}{2}\left(1+\frac{\omega}{k}\right) e^{i k x} \\
\bar{\rho}_{-}(x, t)=\int \frac{d k}{\sqrt{\omega}} a^{+}(k, t) \frac{1}{2}\left(1+\frac{\omega}{k}\right) e^{-i k x}
\end{gathered}
$$

with $\omega \equiv \sqrt{k^{2}+m^{2}}$. The exponential operator and the normal-ordered operator are related by

$$
\exp [i a \rho(x)+i b \bar{\rho}(x)]=\left(: e^{i a \rho(x)+i b \bar{\rho}(x)}:\right)_{m} \exp \left[-\frac{1}{2}\left\langle(a \rho+b \bar{\rho})^{2}\right\rangle\right]
$$

The vacuum expectation values are

$$
\begin{aligned}
\langle\rho \rho\rangle & =\left\langle\rho_{+}(x) \rho_{-}(x)\right\rangle \\
& =\int \frac{d k}{\omega} \theta(|k|-\mu) \frac{1}{4}\left(1-\frac{\omega}{k}\right)^{2} \\
& =\ln \frac{2 \Lambda}{\mu+\sqrt{m^{2}+\mu^{2}}}+\frac{1}{2}\left(\frac{\sqrt{m^{2}+\mu^{2}}}{\mu}-1\right), \\
\langle\bar{\rho} \bar{\rho}\rangle & =\int \frac{d k}{\omega} \theta(|k|-\mu) \frac{1}{4}\left(1+\frac{\omega}{k}\right)^{2} \\
& =\ln \frac{2 \Lambda}{\mu+\sqrt{m^{2}+\mu^{2}}}+\frac{1}{2}\left(\frac{\sqrt{m^{2}+\mu^{2}}}{\mu}-1\right),
\end{aligned}
$$

and

$$
\begin{aligned}
\langle\rho \bar{\rho}\rangle & =\int \frac{d k}{\omega} \theta(|k|-\mu) \frac{1}{4}\left(1-\frac{\omega^{2}}{k^{2}}\right) \\
& =\frac{1}{2}\left(1-\frac{\sqrt{m^{2}+\mu^{2}}}{\mu}\right)
\end{aligned}
$$

where we have introduced an ultraviolet cutoff $\Lambda$ and an infrared cutoff $\mu$. Substituting (A9) into (A8), we obtain

$$
\begin{aligned}
\exp (i a \rho(x) & +i b \bar{\rho}(x))=\left(: e^{i a \rho(x)+i b \bar{\rho}(x)}:\right)_{m} \\
& \times \exp \left[-\frac{a^{2}+b^{2}}{2} \ln \frac{2 \Lambda}{\mu+\sqrt{m^{2}+\mu^{2}}}-\frac{(a-b)^{2}}{4}\left(\frac{\sqrt{m^{2}+\mu^{2}}}{\mu}-1\right)\right] .
\end{aligned}
$$


For $a \neq b$, there is a strong infrared cutoff dependent factor in the exponent. The normal-ordered vertex functions of a finite and a zero reference mass are related by

$$
\begin{aligned}
\left(: e^{i a \rho(x)+i b \bar{\rho}(x)}:\right)_{m} & =\left(\frac{2 \mu}{\mu+\sqrt{\mu^{2}+m^{2}}}\right)^{\frac{a^{2}+b^{2}}{2}} \\
& \times \exp \left[\frac{(a-b)^{2}}{4}\left(\frac{\sqrt{m^{2}+\mu^{2}}}{\mu}-1\right)\right]\left(: e^{i a \rho(x)+i b \bar{\rho}(x)}:\right)_{0} .
\end{aligned}
$$

Vertex operators with two different reference masses are related by

$$
\begin{aligned}
\left(: e^{i a \rho(x)+i b \bar{\rho}(x)}:\right)_{m^{\prime}} & =\left(\frac{\mu+\sqrt{\mu^{2}+m^{2}}}{\mu+\sqrt{\mu^{2}+m^{\prime 2}}}\right)^{\frac{a^{2}+b^{2}}{2}} \\
& \times \exp \left[\frac{(a-b)^{2}}{4 \mu}\left(\sqrt{\mu^{2}+m^{2}}-\sqrt{\mu^{2}+m^{\prime 2}}\right)\right]\left(: e^{i a \rho(x)+i b \bar{\rho}(x)}:\right)_{m}
\end{aligned}
$$

For $a \neq b,(\mathrm{~A} 12)$ contains an infrared dependent factor $\exp \left[(a-b)^{2}\left(m-m^{\prime}\right) / 4 \mu\right]$ as $\mu \rightarrow 0$ which implies that $\left(: e^{i a \rho+i b \bar{\rho}}:\right)_{m}$ defined above is not free from infrared divergence. A possible candidate for a $\mu$-independent vertex operator is

$$
\begin{aligned}
\left(W_{a, b}(x)\right)_{m} & =D_{a, b}\left(: e^{i a \rho(x)+i b \bar{\rho}(x)}:\right)_{m} \\
& \equiv \exp \left[-(a-b)^{2}\left(\sqrt{\mu^{2}+m^{2}}-\mu\right) / 4 \mu\right]\left(: e^{i a \rho+i b \bar{\rho}}:\right)_{m} \\
& =\left(\frac{2 \mu}{\mu+\sqrt{\mu^{2}+m^{2}}}\right)^{\frac{a^{2}+b^{2}}{2}}\left(: e^{i a \rho+i b \bar{\rho}}:\right)_{0} .
\end{aligned}
$$

These new normal-ordered operators have infrared independent transformation law at $m, m^{\prime}>>\mu$

$$
\left(W_{a, b}(x)\right)_{m^{\prime}}=\left(\frac{m}{m^{\prime}}\right)^{a^{2}+b^{2}}\left(W_{a, b}(x)\right)_{m}
$$

These $\left(W_{a, b}(x)\right)_{m}$ provide $\mu$-independent components for the construction of conserved currents in the framework of a finite reference mass. Note that $\left(W_{a, b}(x)\right)_{0}=\left(: e^{i a \rho+i b \bar{\rho}}:\right)_{0}$ is the vertex operator defined w.r.t. zero mass, as used extensively in ref. 4.

In ref.4, we have shown that the following currents are conserved $\left(k_{0}=\mu e^{\gamma}\right)$,

$$
\begin{gathered}
\left(J_{+}(x, t)\right)_{0}=\left(A_{\frac{2}{\beta}}(x, t)\right)_{0}+\frac{\pi \lambda_{0}}{2 \mu^{2} e^{2 \gamma}}\left(W_{\frac{2}{\beta}-\beta,-\beta}(x, t)\right)_{0}, \\
\left(J_{-}(x, t)\right)_{0}=\frac{\pi \lambda_{0}}{2 \mu^{2} e^{2 \gamma}} \frac{\beta^{2}}{2-\beta^{2}}\left(W_{\frac{2}{\beta}-\beta,-\beta}(x, t)\right)_{0}, \\
\partial_{-}\left(J_{+}\right)_{0}+\partial_{+}\left(J_{-}\right)_{0}=0 .
\end{gathered}
$$


In terms of $\left(W_{a, b}(x)\right)_{m}$, we introduce

$$
\begin{aligned}
\left(J_{+}(x, t)\right)_{m} & =\left(A_{\frac{2}{\beta}}(x, t)\right)_{m}+\frac{2 \pi \lambda_{m}}{\left(\mu+\sqrt{\mu^{2}+m^{2}}\right)^{2} e^{2 \gamma}}\left(W_{\frac{2}{\beta}-\beta,-\beta}(x, t)\right)_{m} \\
& =\left(\frac{2 \mu}{\mu+\sqrt{\mu^{2}+m^{2}}}\right)^{\frac{2}{\beta}}\left(J_{+}(x, t)\right)_{0} \\
\left(J_{-}(x, t)\right)_{m} & =\frac{2 \pi \lambda_{m}}{\left(\mu+\sqrt{\mu^{2}+m^{2}}\right)^{2} e^{2 \gamma}} \frac{\beta^{2}}{2-\beta^{2}}\left(W_{\frac{2}{\beta}-\beta,-\beta}(x, t)\right)_{m} \\
& =\left(\frac{2 \mu}{\mu+\sqrt{\mu^{2}+m^{2}}}\right)^{\frac{2}{\beta}}\left(J_{+}(x, t)\right)_{0}
\end{aligned}
$$

and obtain

$$
\partial_{-}\left(J_{+}\right)_{m}+\partial_{+}\left(J_{-}\right)_{m}=0
$$

Note that the infrared factor $\exp \left[-(a-b)^{2}\left(\sqrt{m^{2}+\mu^{2}}-\mu\right) / 4 \mu\right]$ is $\mathrm{x}$-independent, and is the same for all terms of $J_{+}$and $J_{-}$. Hence, (A20) follows from (A17).

In the following, we shall derive the above current conservation entirely in the framework of a finite reference mass $m$. The equal-time commutation relations among $\rho$ and $\bar{\rho}$ are still given by (2.5), independent of the interaction and the reference mass. However, the separation of $\rho$ into $\rho_{-}$ and $\rho_{+}$depends on the reference mass, and so are the equal-time commutation relations among $\rho_{ \pm}$and $\bar{\rho}_{ \pm}$. They are

$$
\begin{aligned}
& {\left[\rho_{+}(x, t), \rho_{-}(y, t)\right] }=\int \frac{d k}{\omega} \frac{1}{4}\left(1-\frac{\omega}{k}\right)^{2} e^{i k(x-y)} \\
&= K_{0}(m|x-y|)+\frac{1}{2} M(m|x-y|)-\frac{i \pi}{2} \epsilon(x-y) \\
& {\left[\bar{\rho}_{+}(x, t), \bar{\rho}_{-}(y, t)\right]=K_{0}(m|x-y|)+\frac{1}{2} M(m|x-y|)+\frac{i \pi}{2} \epsilon(x-y) } \\
&=-\frac{1}{2} M(m(x-y))-\frac{i \pi}{2} \\
& {\left[\rho_{+}(x, t), \bar{\rho}_{-}(y, t)\right]=\int \frac{d k}{\omega} \frac{1}{4}\left(1-\frac{\omega^{2}}{k^{2}}\right) e^{i k(x-y)} } \\
& {\left[\rho_{-}(x, t), \bar{\rho}_{+}(y, t)\right]=\frac{1}{2} M(m(x-y))-\frac{i \pi}{2} }
\end{aligned}
$$

where $K_{0}(z)$ is a modified Bessel function, and $M(m(x-y))$ is defined as 


$$
M(m(x-y)) \equiv \frac{1}{2} \int \frac{d k}{\omega} \frac{m^{2}}{k^{2}} e^{i k(x-y)}
$$

and

$$
\frac{d^{2} M(z)}{d z^{2}}=-K_{0}(z)
$$

The additional $-i \pi / 2$ terms in (A.21c) and (A.21d) come from $k=0$ mode as required by the nonsymmatric definition of $\rho$ and $\bar{\rho}$ relative to $x= \pm \infty . M(m(x-y))$ contains an additive infrared cutoff dependent constant, $C(m / \mu)=\left(\sqrt{m^{2}+\mu^{2}}-\mu\right) / \mu$. This infrared dependent constant is the origin of the required infrared factor in $\left(W_{a, b}\right)_{m}$.

In our calculation, we only need commutators between $\rho_{ \pm}, \bar{\rho}_{ \pm}$and $\phi_{ \pm}$. These commutators are free of infrared divergences,

$$
\begin{aligned}
{\left[\rho_{+}(x, t), \phi_{-}(y, t)\right] } & =\int \frac{d k}{\omega} \frac{1}{2}\left(1-\frac{\omega}{k}\right) e^{i k(x-y)} \\
& =K_{0}(m|x-y|)-i \pi \theta(x-y) \\
{\left[\rho_{-}(x, t), \phi_{+}(y, t)\right] } & =-K_{0}(m|x-y|)-i \pi \theta(x-y) \\
{\left[\bar{\rho}_{+}(x, t), \phi_{-}(y, t)\right] } & =K_{0}(m|x-y|)+i \pi \theta(x-y) \\
{\left[\bar{\rho}_{-}(x, t), \phi_{+}(y, t)\right] } & =-K_{0}(m|x-y|)+i \pi \theta(x-y) .
\end{aligned}
$$

To derive the conservation law, we follow the method described in sec. 3. The Heisenberg eq. gives

$$
\begin{aligned}
\partial_{-}\left(W_{\frac{2}{\beta}, 0}(x, t)\right)_{m} & =-\frac{i}{2}\left[\left(W_{\frac{2}{\beta}, 0}(x, t)\right)_{m}, \int d y V(\phi(y))\right] \\
& =i \frac{\lambda_{m}}{4} \int d y\left[\left(W_{\frac{2}{\beta}, 0}(x)\right)_{m},\left(W_{\beta, \beta}(y)+W_{\beta,-\beta}(y)\right)_{m}\right] \\
& =\frac{\lambda_{m}}{4} \int d y\left\{D_{\frac{2}{\beta}, 0}\left(: e^{\frac{2 i}{\beta} \rho(x, t)+i \beta \phi(y, t)}:\right)_{m}\right. \\
& \left.\times\left[e^{-2\left[\rho_{+}(x, t), \phi_{-}(y, t)\right]}-e^{2\left[\rho_{-}(x, t), \phi_{+}(y, t)\right]}\right]+(\beta \leftrightarrow-\beta)\right\} \\
& =\frac{\lambda_{m}}{4} \int d y\left\{D_{\frac{2}{\beta}, 0}\left(: e^{\frac{2 i}{\beta} \rho(x, t)+i \beta \phi(y, t)}:\right)_{m}\right. \\
& \left.\times\left[e^{-2 K_{0}(m|x-y|)+2 i \pi \theta(x-y)}-e^{-2 K_{0}(m|x-y|)-2 i \pi \theta(x-y)}\right]+(\beta \leftrightarrow-\beta)\right\} .
\end{aligned}
$$


For $x \neq y$, the integrand vanishes. Thus, all the contribution comes from the infinitesimal region around $y=x$. Near $y=x$, we have

$$
\begin{aligned}
K_{0}(m|x-y|) & =-\gamma-\ln \left(\frac{1}{2} m|x-y|\right)+0\left((x-y)^{2}\right) \\
{\left[\bar{\rho}_{-}(x, t), \phi_{+}(y, t)\right] } & =\gamma+\ln \left(\frac{1}{2} m|x-y|\right)-i \pi \theta(x-y)+0\left((x-y)^{2}\right) \\
& =\ln (-i \bar{m}(x-y+i \epsilon))-\frac{i \pi}{2}+0\left((x-y)^{2}\right)
\end{aligned}
$$

where

$$
\bar{m} \equiv \frac{1}{2} m e^{2}
$$

Eq. (A.27) has the same short distance structure as those obtained from (2.7) - (2.9) of ref. 4 with the replacement of $k_{0}$ by $\bar{m}$. This is in fact true for other terms in the commutators, and the derivation of new currents in ref. 4 follow through. This leads to the construction of a conserved current which has the same structure of (A.15) (A.16), ((3.11) and (3.12) of ref. 4) with the replacement of $\left(W_{a, b}\right)_{0}$ by $\left(W_{a, b}\right)_{m}, \lambda_{0}$ by $\lambda_{m}$, and $k_{0}$ by $\bar{m}$, giving (at $m$ finite and $\mu \rightarrow 0$ ),

$$
\begin{aligned}
& \left(J_{+}(x, t)\right)_{m}=\left(W_{\frac{2}{\beta}, 0}(x, t)\right)_{m}+\frac{2 \pi \lambda_{m}}{m^{2} e^{2 \gamma}}\left(W_{\frac{2}{\beta}-\beta,-\beta}(x, t)\right)_{m} \\
& \left(J_{-}(x, t)\right)_{m}=\frac{2 \pi \lambda_{m}}{m^{2} e^{2 \gamma}} \frac{\beta^{2}}{2-\beta^{2}}\left(W_{\frac{2}{\beta}-\beta,-\beta}(x, t)\right)_{m}
\end{aligned}
$$

as desired.

\section{APPENDIX B: CONTRIBUTIONS FROM SHORT DISTANCE SINGULARIES}

Consider the integral

$$
I=\lim _{\epsilon \rightarrow 0} \int_{-L}^{L} d x\left(x^{2}+\epsilon^{2}\right)^{p}\left((x+i \epsilon)^{n}-(x-i \epsilon)^{n}\right) C(x)
$$

where $n$ is a positive integer, $p$ is real and $C(x)$ is a nonsingular function. We see that the integrand vanishes as $\epsilon \rightarrow 0$ for any $x \neq 0$. Hence the integral will receive nonzero contributions, if any, only from possible singularities at $x=0$. We restrict the range of $x$ to be in $[-L, L]$ with $L^{-1} \sim \mu$, the 
infrared mass we have used throughout the paper. We evaluate this integral with the understanding that $\epsilon \rightarrow 0$ first, and then $L$ can tend to infinity. Expand

$$
\begin{gathered}
C(x)=\sum_{m=0}^{\infty} x^{m} C_{m}, \\
(x+i \epsilon)^{n}=\sum_{r=0}^{n} x^{n-r}(i \epsilon)^{r} \frac{n !}{r !(n-r) !},
\end{gathered}
$$

and change variables to $y=x / \epsilon$. Then,

$$
I=\lim _{\epsilon \rightarrow 0} \sum_{m=0}^{\infty} \sum_{\text {odd } r}^{n} \epsilon^{2 p+1+n+m} K_{m r} \int_{-L / \epsilon}^{L / \epsilon} d y\left(1+y^{2}\right)^{2 p} y^{n+m-r}
$$

where

$$
K_{m r}=\frac{2(i)^{r} n !}{r !(n-r) !} C_{m}
$$

As $\epsilon \rightarrow 0$, the limits of the y-integration tend towards infinity. For large $y$, the y-integrand behaves as $y^{2 p+n+m-r}$. Let $N$ be the largest (positive or negative) integer less than $(-2 p-n-1)$. Then for $m-r \leq N$ the y-integral converges even when $\epsilon \rightarrow 0$, whereas, for $m-r>N$, it behaves as $(L / \epsilon)^{2 p+n+m-r+1}$. Hence $I$ has the form

$$
I=\lim _{\epsilon \rightarrow 0}\left[\sum_{\text {odd } r}^{n} \sum_{m}^{m-r \leq N} \epsilon^{2 p+n+m+1} A_{r m}+\sum_{m}^{m-r>N} \sum_{o d d r}^{n} \epsilon^{r} B_{r m}\right]
$$

where $A_{r m}$ and $B_{r m}$ represent the result of y-integrals multiplied by finite factors like $K_{r m}$ in (B.3). For any finite $L$, as $\epsilon \rightarrow 0$, the second term vanishes since $r$ is positive. Turning to the first term, notice that the y-integrals in (B.2) vanish by $y \leftrightarrow-y$ symmetry unless $n+m-r$ is even. Since $r$ is odd anyway, this requires $n+m$ odd. Thus, for even (odd) $n$, the lowest value of $m$ that contributed is one (zero). Hence we conclude, for different cases:

(a) $n$ odd

$$
\begin{array}{lll}
\text { (i) } \quad \text { when } 2 p+n+1>0 & I=0 \\
\text { (ii) } \quad \text { when } 2 p+n+1=0 & I=\text { finite } \\
\text { (iii) } \quad \text { when } 2 p+n+1<0 & I \text { diverges as } \epsilon \rightarrow 0 .
\end{array}
$$


Notice that in cases (ii) and (iii) above, there will exist some finite nonzero $A_{r m}$, satisfying $m-r \leq$ $N$, provided the function $C(x)$ and its first derivative are nonzero at $x=0$.

(b) $n$ even
(i) when $2 p+n+2>0 \quad I=0$
(ii) when $2 p+n+2=0 \quad I=$ finite
(iii) when $2 p+n+2<0 \quad I$ diverges as $\epsilon \rightarrow 0$. 


\section{REFERENCES}

* Address after December 1, 1993: School of Physical Sciences, Jawaharlal Nehru University, New Delhi 110067 India.

[1] A. B. Zamolodchikov, Int. J. Mod. Phys. A4, 4235 (1989); ibid A3, 743 (1988).

[2] D. Bernard and A. LeClair, Comm. Math. Phys. 142, 99 (1991); A. LeClair, Cornell Univ. preprint CLNS 93/1220, hep-th/9305110, (1993).

[3] R. K. Kaul and R. Rajaraman, Int. J. Mod. Phys. A8, 1815 (1993).

[4] S. J. Chang and R. Rajaraman, Phys. Lett. B313, 59 (1993).

[5] S. Coleman, Phys. Rev. D11, 2088 (1975).

[6] S. Mandelstam, Phys. Rev. D11, 3026 (1975).

[7] B. Schroer and T. Truong, Phys. Rev. D15, 1684 (1977). 